\title{
The needle in the haystack: where to look for more isolated cooling neutron stars
}

\author{
B. Posselt ${ }^{1,2,3}$, S. B. Popov ${ }^{4}$, F. Haberl ${ }^{1}$, J. Trümper ${ }^{1}$, R. Turolla ${ }^{5}$, and R. Neuhäuser ${ }^{2}$
}

\author{
1 Max-Planck-Institut für extraterrestrische Physik, Postfach 1312, 85741 Garching, Germany \\ 2 Astrophysikalisches Institut und Universitäts-Sternwarte, Schillergäßchen 2-3, 07745 Jena, Germany \\ 3 Observatoire Astronomique de Strasbourg, 11 rue de l' Universite, 67000 Strasbourg, France \\ e-mail: bposselt@cfa.harvard.edu \\ 4 Sternberg Astronomical Institute, Universitetski pr. 13, 119991 Moscow, Russia \\ e-mail: polar@sai.msu.ru \\ 5 University of Padua, Department of Physics, via Marzolo 8, 35131 Padova, Italy
}

Received 7 August 2007 / Accepted 29 January 2008

\section{ABSTRACT}

\begin{abstract}
Context. Isolated, cooling neutron stars with thermal X-ray emission remain rarely-detected objects despite many searches investigating the ROSAT data.

Aims. We simulate the population of close-by young cooling neutron stars to explain the current observational results. Given the inhomogenity of the neutron star distribution on the sky, it is particularly interesting to identify promising sky regions with respect to ongoing and future searches.

Methods. Applying a population synthesis model, the inhomogenity of the progenitor distribution and the inhomogenity of the X-ray absorbing interstellar medium are considered for the first time. The total number of observable neutron stars is derived with respect to ROSAT count rates. In addition, we present sky maps of neutron star locations, and discuss age and distance distributions of the simulated neutron stars. Implications for future searches are discussed.

Results. With our advanced model, we can succesfully explain the observed $\log N-\log S$ distribution of close-by neutron stars. Cooling neutron stars will be most abundant in the directions of rich OB associations. We expect new candidates to be identified behind the Gould Belt, in particular in the Cygnus-Cepheus region. We expect them to be on average younger and hotter than the known population of isolated, cooling neutron stars. In addition, we propose to use data on runaway stars to search for more radio-quiet, cooling neutron stars.
\end{abstract}

Key words. stars: evolution - stars: neutron - X-rays: stars

\section{Introduction}

More than 10 years after the discovery of its brightest member RX J1856-3754 (Walter \& Matthews 1997), the small group of seven radio-quiet isolated neutron stars (NSs) detected by the ROSAT satellite have gained an important place in the rich zoo of compact objects. Together with Geminga and several close-by young radio pulsars, these objects form the local population of cooling NSs. Studies of this group of sources already provided a wealth of information on NSs physics (see, e.g., Haberl 2007; Page et al. 2007; Zane 2007, for recent reviews).

Since 2001 the number of known close-by, radio-quiet NSs has not been growing despite all attempts to identify (mainly in the ROSAT data) new candidates. Partly, this is due to the fact that all the searches cited above were blind, i.e., not necessarily targeted to promising sky regions. To advance the identification of new nearby, cooling NSs, it is necessary to perform a realistic modeling of this population. The seven ROSAT radio-quiet NSs form the main part of the known local population of cooling NSs. For these seven sources, we use the term Magnificent Seven. There is still no general term to address the whole population to which these sources belong (terms such as XDINS - X-ray dim isolated NSs, DINS - dim isolated NSs, RINS - ROSAT (or radio quiet) isolated NSs, are used sometimes by different authors). In this paper, we use the term ICoNSs (Isolated Cooling
NSs). In our opinion, this variant better reflects the nature of these sources than, for example, XDINS, as many of them are not dim in X-rays. Finally, we call coolers all NSs, whose residual thermal emission can be detected. Besides the radio-quiet NSs, such sources can be also known as normal radio pulsars, or they may demonstrate some kind of radio emission or even $\gamma$-ray emission distinct from classical pulsars. In this study we diregard the possibility that an object shows some activity in addition to thermal surface emission. The only crucial point is the detection of X-ray emission related to the cooling of initially-hot compact objects.

We used the population synthesis (PS) approach to investigate the population of close-by young cooling NSs. There are two main versions of this method (Fritze-v. Alvensleben 2000). One, which is sometimes called empirical population synthesis, is a kind of top-down approach, when objects, for which only integral characteristics are available, are studied. Numerical models are applied to reproduce observed properties of such sources with different sets of subpopulations that constitute an object class. For example, the stellar content of a galaxy can be derived by modeling its integral spectra.

Here, we focus on the second - bottom-up - approach, often called evolutionary synthesis. In this case, the evolution of a population of objects is modeled from their birth based on defined initial distributions of parameters and some equations that 
describe evolution of these distributions in time. We use this second technique for the following reasons. At first, if initial parameters or/and evolutionary laws for some kind of sources are not well known, then this kind of PS can be applied to test hypotheses about these uncertain properties with a comparison between modeled and observed populations. Determination of the initial parameters of radio pulsars are a good example here (Faucher-Giguère \& Kaspi 2006). Next, even if the initial distributions and their evolution are known, but at the moment just a small part of the population - a tip of an iceberg - is observed, then PS calculations can be used to predict properties of the unobserved faint part of the population, and to plan a search strategy to identify new members. A more detailed review of the PS technique can be found, for example, in Popov \& Prokhorov (2004).

In this paper, an upgraded population synthesis model is discussed for the population of close-by $(<3 \mathrm{kpc})$ isolated NSs, which can be observed via their thermal emission in soft X-rays. Previously, we applied our models to confirm the link between the Magnificent Seven and the Gould Belt (Popov et al. 2003), and to test theories of thermal evolution of NSs (Popov et al. 2006). The major interest of the present study is to understand how to find more objects of this type. After the description of the PS model ingredients in Sect. 2, we present our results $\log N-\log S$-curves, age and distance diagrams as well as sky maps of expected NS locations in Sect. 3, and discuss possible applications in Sect. 3.4. An outlook concerning the search for ICoNSs is given in Sect. 3.5 Finally, we give our conclusions in Sect. 4.

\section{The new population synthesis model}

The main physical ingredients that constitute our population synthesis model are the following:

(A) the initial NS spatial distribution and the NS birth rate;

(B) the kick velocity distribution of the NSs;

(C) the Galactic gravitational potential;

(D) the distribution of NS masses, in the following: NS mass spectrum;

(E) the NS cooling curve;

(F) the NS surface emission in X-rays;

(G) the interstellar absorption of X-rays;

(H) the properties of the X-ray detector.

At each time step in the simulation, we consider at the same time, eight different NS masses with corresponding cooling curves, following each NS on its trajectory through space and time until it is too faint to be observed. The overall result of, e.g., $50000 \times 8$ simulated NS evolutionary tracks from birth till the time when the temperatures falls below $10^{5} \mathrm{~K}$, is normalized by the mass distribution as well as by birth rates (see below for both). Ingredients B, C, E, and F are unchanged with respect to our previous studies (Popov et al. 2003, 2005, 2006, hereafter Papers I, II, III), so we just briefly comment on them below. The ingredients $A, D, G$, and $\mathrm{H}$ - the initial spatial distribution, the NS mass spectrum, the interstellar absorption, and the X-ray detector properties - are modified, and they are described in subsections below in more detail.

For the kick velocity distribution (B) of newborn neutron stars, we use the distribution proposed by Arzoumanian et al. (2002), as in all our previous studies. This is a bimodal distribution, consisting of two Maxwellians with most probable velocities $V_{\text {peak } 1} \approx 127 \mathrm{~km} \mathrm{~s}^{-1}$ and $V_{\text {peak } 2} \approx 707 \mathrm{~km} \mathrm{~s}^{-1}$, the average velocity is $\approx 527 \mathrm{~km} \mathrm{~s}^{-1}$. Recently, this assumption was criticized. A single-component distribution with $V_{\text {peak }} \approx 400 \mathrm{~km} \mathrm{~s}^{-1}$ was advocated by Hobbs et al. (2005), and by Faucher-Giguère \& Kaspi (2006) who accepted a non-Maxwellian velocity distribution. However, since all the objects under study are very young and are still relatively close to their birth place, the exact shape of the velocity distribution does not have a strong influence on our final results.

The calculation of NS trajectories in the Galactic gravitational potential (C) follows the methods presented in Papers I and II. As before, we use the axisymmetric Miyamoto-Nagai (1975) potential with disk, buldge, and halo contributions. This potential (with some modifications) was already actively used in NS calculations by different authors in the 1990s (see, for example, Blaes \& Madau 1993, and references therein). Again, it is noted that since the NS are young the use of a very precise multicomponent potential is not necessary. Thus, we also neglect the Galactic bar, which extends to a maximum Galactocentric distance of $3.5 \mathrm{kpc}$ (e.g. Pichardo et al. 2004) because its influence is very small for the relatively nearby objects of interest.

The thermal evolution of NSs is one of the main ingredients of the study. There are strong uncertainties in this topic. Sets of curves have been calculated by many research groups for different models of NS interiors (see reviews, e.g., by Page \& Reddy 2006; or Yakovlev \& Pethick 2004). In the following, we consider only hadron stars and use cooling curves (E) corresponding to those marked as model I in Paper III. This cooling model includes the following important ingredients. Superfluid gaps are taken according to Takatsuka \& Tamagaki (2004). Medium modifications of the neutrino processes are taken into account. Formation of the pion condensate is possible. The relation between temperatures of the outermost core layer and the surface is taken from Blaschke et al. (2004). No additional heating is used.

In our calculations, we do not account for atmospheric reprocessing of the thermal radiation, and assume that the emitted spectrum is a pure blackbody (see a detailed description of the modeling of the observed neutron star thermal emission in Zavlin 2007). Unfortunately, the distribution of the chemical compositions of atmospheres is not known for the whole population of young NSs. Whether or not the atmospheric composition changes in time is also unclear. As an additional parameter one needs to consider further the (unknown) magnetic field strength and its effects on the atmosphere. So, the available data are not complete enough and the statistics are too small to consider atmospheres in population synthesis studies.

However, isotropic blackbody emission is a simplification since there is growing evidence that the thermal X-ray emission observed in case of the Magnificent Seven actually comes from hot spots and not from the whole NS surface (e.g., Haberl et al. 2006; Schwope et al. 2005; Trümper et al. 2004). This is expected to influence not only the cooling times, but also the detection probabilities. But the overall quantitative effect is currently unclear as at first it is necessary to include nonuniform heat transport in the outer NS layers into cooling curves calculations self-consistently, for example, in the way it is discussed in Aguilera et al. (2007). Strong toroidal magnetic fields produce hot spots and can keep them hot over a longer time than the usual lifetime expected from the previous cooling models (Page et al. 2007).

Since the hot spot of a neutron star will have a higher temperature than in the case of isotropic cooling, its detection would be less affected by interstellar absorption. The fact that these effects are not accounted for by our applied cooling curves leads 
us to underestimate the number of observable neutron stars. On the other hand, Yakovlev \& Pethick (2004) have pointed out that at high magnetic field strength the break of the cooling curves (marking the transition from neutrino to photon cooling) shifts toward lower ages, viz. from $\sim 10^{6}$ years at $B \sim 0 \mathrm{G}$ to a few times $10^{5}$ years for magnetic fields typical for the Magnificent Seven, $B \sim 10^{13}-10^{14} \mathrm{G}$. Neglecting this effect will lead to an overestimate of the number of observable neutron stars with ages of $(0.5-1) \times 10^{6}$ years where some of the Magnificent Seven are found. Magnetic field decay and the additional heating due to it (Aguilera et al. 2008) can further increase the number of observable NSs. We cannot make a firm prediction about the net change to the $\log N-\log S$ that is caused by these effects as they are partly counterbalancing. However, we believe that the blackbody estimate of the count rate $S$ is reliable within a factor of $\sim 2$.

The soft X-ray spectra of objects under consideration are well fitted by the blackbody shape, and, more importantly, the cooling curves used here were calculated under the assumption of blackbody emission of the whole NS surface, and cooling curves for nonuniform emission are currently not publicly available. We, therefore, stick to the cooling curves of Paper III and the assumption of spherical symmetric blackbody emission, and apply them as a reference model to study the main aspect of this paper - the influence of the inhomogeneous distributions of neutron star birth places and interstellar absorptions in the Galaxy.

\subsection{Initial spatial distribution of NSs}

Following the results of previous investigations (Paper I), we accept as established that the population of nearby NSs is genetically related to the Gould Belt. The contribution of the Gould Belt dominated the production of compact remnants in the solar proximity over the past $\sim 30 \mathrm{Myr}$ (see Pöppel 1997 for a detailed description of the Gould Belt structure). About two thirds of massive stars in the $\sim 600 \mathrm{pc}$ around the Sun belong to the Gould Belt (Torra et al. 2000).

In Paper I and II we considered that NSs are born either in the Belt or in the Galactic disk, both treated as infinitesimally thin, disk-like structures, with a uniform spatial distribution. The total NS birth rate in the Galactic disk (the whole area inside $3 \mathrm{kpc}$ ), and the rate in the Gould Belt alone, were taken to be $270 \mathrm{Myr}^{-1}$ and $20 \mathrm{Myr}^{-1}$, respectively. The first value corresponds to the value inside $1 \mathrm{kpc}$ by Tammann et al. (1994). The latter value is from Grenier (2000), and appears to be in reasonable agreement with the historical supernova rate inside $1 \mathrm{kpc}$, as estimated by Tammann et al. (1994).

Here, the previously simplified progenitor distribution is significantly upgraded by adopting a more realistic description of the massive star geography in the solar neighborhood. In particular, in the innermost $500 \mathrm{pc}$ from the Sun, the initial spatial distribution of NSs is assumed to follow the presently-observed distribution of massive stars. Outside the well-known $500 \mathrm{pc}$ and up to $3 \mathrm{kpc}$, NSs are born either in the Galactic disc, assumed to be exponential both in $z$ and $R$ directions, or originate from known, rich $\mathrm{OB}$ associations.

\subsubsection{Inside 500 pc}

For the stars inside 500 pc we use the Hipparcos data on massive stars (Perryman \& ESA 1997). Our assumption is that the present day distribution of massive stars well represents their distribution in the last 1-2 million years.
Table 1. OB associations inside $500 \mathrm{pc}$ and their ages (from de Zeeuw et al. 1999). For Ori OB1 the age proposed for its older part Ori OB1A is taken.

\begin{tabular}{lc}
\hline \hline OB association & Age [Myr] \\
\hline Upper Scorpius & $4-6$ \\
Upper Centaurus Lupus & $12-14$ \\
Lower Centaurus Crux & $9-11$ \\
Vel OB2 & $6-10$ \\
Trumpler 10 & $15-30$ \\
Collinder 121 & $4-6$ \\
Ori OB1 & $9.5-13.3$ \\
Per OB2 & $3-7$ \\
$\alpha$ Persei (Per OB3) & $25-50$ \\
Cas-Tau & $25-50$ \\
Lac OB1 & $2-25$ \\
Cep OB2 & $5-10$ \\
Cep OB6 & $25-50$ \\
\hline
\end{tabular}

All the 570 classified B2-O8 stars with known parallaxes $>0$.'002 are considered. According to the birthrates above, in our model 27 (out of 270) NS producing supernova events per Myr are assumed to be related to this population. This number should be compared with $20 \mathrm{NSs}$ assumed to be born in the entire Gould Belt in 1 Myr in our old model. The parameters of the new model roughly lie in between the old one for $R_{\text {Belt }}=300$ and $500 \mathrm{pc}$.

In the code, the initial position of each newborn NS coming out of this population coincides with the position of one of these 570 Hipparcos stars. This assumes that the spatial distribution of the massive main sequence stars does not change significantly over the NS evolutionary timescale ( $\approx 1 \mathrm{Myr}$ ). A progenitor star is chosen randomly, but we tried to account for the probability for each particular star to explode. Since for each star the spectral type is known, one can roughly estimate its mass. The mass interval corresponding to each spectral type gives us an estimated range for its total lifetime. If one has no information about the age of the star then the probability is assumed to depend only on the star's full lifetime: the probability is higher for more massive stars as they are shorter lived (actually, the probability to explode is inversely proportional to the lifetime of the star).

As noted above, the mass interval corresponding to each spectral class is converted into an approximate limiting lifetime: $T_{\mathrm{LM}}$ is the longest lifetime for the lowest mass, and $T_{\mathrm{HM}}$ is the shortest lifetime for the high mass end of the particular mass interval. If the star is known to belong to an OB association, we also consider the age of the OB association. The ages of the OB associations are shown in Table 1; they are taken from de Zeeuw et al. (1999). In several cases, de Zeeuw et al. (1999) give just one number (not a range) as an estimate of an age. Of course, these numbers have some uncertainty. To account for this we added an uncertainty $( \pm 1 \mathrm{Myr})$ in all those cases. By this, we have for all $\mathrm{OB}$ associations in the table $T L_{\mathrm{OB}}$ and $T U_{\mathrm{OB}}$, a lower and upper age limit for the $\mathrm{OB}$ association, respectively.

The probability for a progenitor star to explode (and so to contribute to NS production in our model) is estimated as follows.

(i) If $T_{\mathrm{HM}}>T U_{\mathrm{OB}}$, then the probability of a star exploding is zero, as the shortest estimated lifetime is longer than the age of the OB association. Such stars do not contribute to NS production in our model, i.e., no compact objects could have been born at their locations because the OB association is too young. 

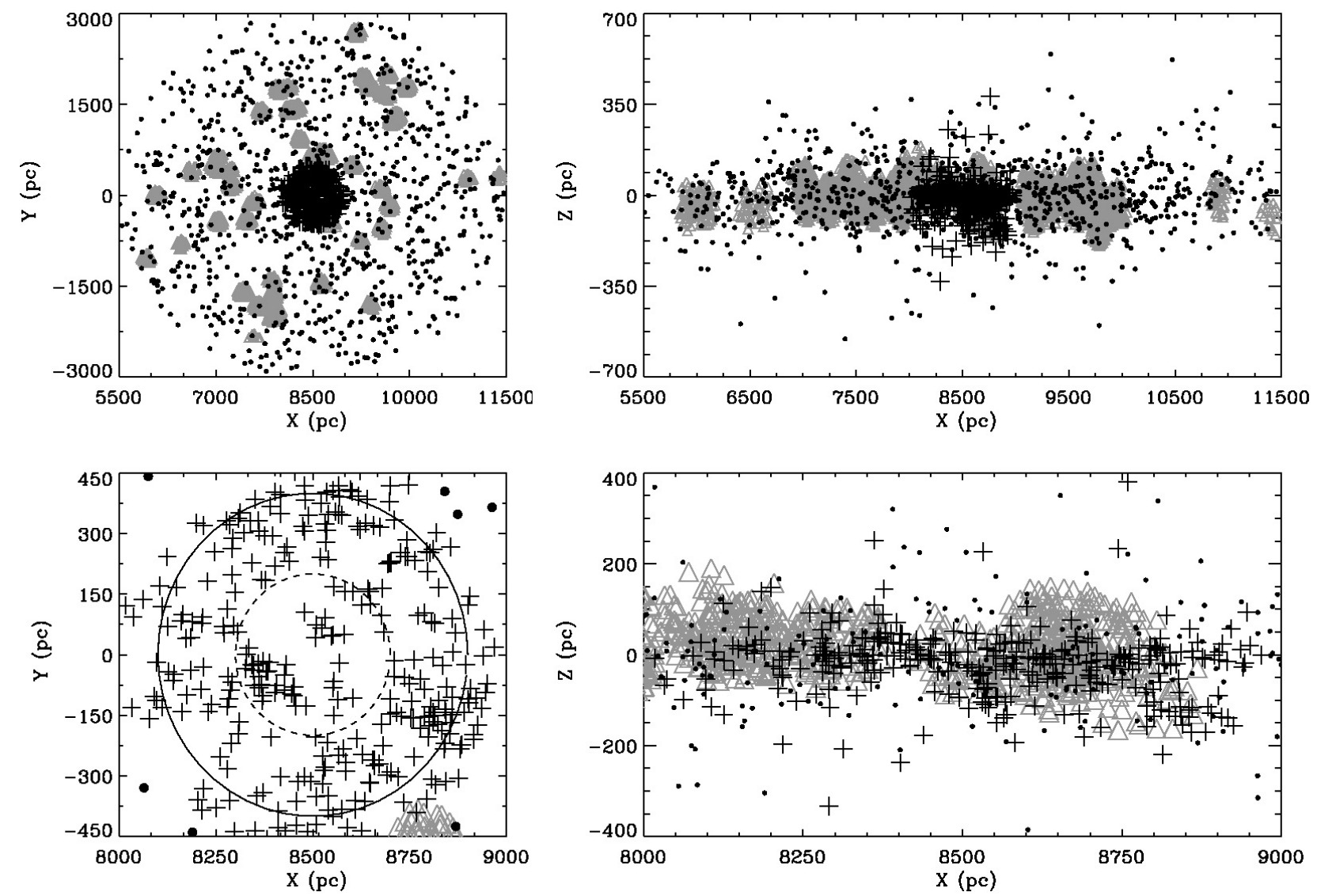

Fig. 1. The new NS progenitor distribution. The grey triangles represent the stars in the OB associations. Crosses stand for the considered Hipparcos stars inside $500 \mathrm{pc}$. Small circles mark random stars in the Galactic disk $(500<d<3000 \mathrm{pc})$. Outside $500 \mathrm{pc}$ from the Sun two populations are visible: NSs formed in OB associations, and NSs formed in the field. Bottom panels show the projected distribution X-zoomed to 500 pc around the Sun. Two circles on the bottom left panel are plotted with radii of 200 and $400 \mathrm{pc}$ from the Sun.

(ii) Conversely, if $T_{\mathrm{LM}}<T L_{\mathrm{OB}}$, then the probability is inversely proportional to the longest lifetime, $T_{\mathrm{LM}}$, of the star. This probability is also used for stars not belonging to $\mathrm{OB}$ associations within a $500 \mathrm{pc}$ distance.

(iii) If $T_{\mathrm{HM}}<T L_{\mathrm{OB}}$, but $T_{\mathrm{LM}}>T U_{\mathrm{OB}}$, then the probability is inversely proportional to $\left(T U_{\mathrm{OB}}-T L_{\mathrm{OB}}\right)$, as one has to consider overlapping time scales.

(iv) If $T_{\mathrm{HM}}<T L_{\mathrm{OB}}$ and $T L_{\mathrm{OB}}<T_{\mathrm{LM}}<T U_{\mathrm{OB}}$, then the probability of exploding is inversely proportional to $\left(T_{\mathrm{LM}}-\right.$ $\left.T L_{\mathrm{OB}}\right)$.

(v) If $T L_{\mathrm{OB}}<T_{\mathrm{HM}}<T U_{\mathrm{OB}}$ and $T_{\mathrm{LM}}>T U_{\mathrm{OB}}$, then the probability is inversely proportional to $\left(T U_{\mathrm{OB}}-T_{\mathrm{HM}}\right)$.

(vi) If $T L_{\mathrm{OB}}<T_{\mathrm{HM}}$, but $T_{\mathrm{LM}}<T U_{\mathrm{OB}}$, then the probability of exploding is inversely proportional to the minimum time value $\left(T_{\mathrm{LM}}-T_{\mathrm{HM}}\right)_{\mathrm{MIN}}=1.44 \mathrm{Myr}$, which was obtained for the spectral type B0, corresponding to the mass interval $\left[15 M_{\odot}, 18 M_{\odot}\right]$.

To summarize, the initial spatial distribution of NSs inside $500 \mathrm{pc}$ in our model follows the distribution of Hipparcos massive stars weighted by their explosion probabilities as estimated above. Outside $500 \mathrm{pc}$ this weighting is not applied.

\subsubsection{Outside $500 \mathrm{pc}$, up to $3 \mathrm{kpc}$}

Most of the NSs in our model, 200 out of 270 per Myr, are born in one of $49 \mathrm{OB}$ associations outside $500 \mathrm{pc}$ and up to $3 \mathrm{kpc}$. The radii of the association extents are taken to be equal to $100 \mathrm{pc}$.
This is a safe upper limit on their sizes, as usually diameters are about 80 pc, and up to 200 pc (Mel'nik \& Efremov 1995). The probability to be born inside this radius is uniform. We assume that the number of stars with known photometry roughly reflects the total number of stars in the cluster. The probability to be born in each cluster is proportional to this quantity. The data on the $49 \mathrm{OB}$ associations are taken from the catalogue by Blaha $\&$ Humphreys (1989) (see also Mel'nik \& Efremov 1995). Note, that distances to the associations are reduced by $20 \%$, according to conclusions by Dambis et al. (2001).

Finally, out of the total 270 NSs, 43 are supposed to be born during 1 Myr in the Galactic disk outside 500 pc from the Sun, but inside $3 \mathrm{kpc}$. Their positions in the disk are calculated randomly. The disk is exponential both in $R$ and $Z$. For the disk $Z$-scale we take the value $100 \mathrm{pc}$. For the radial scale we take the value $3 \mathrm{kpc}$ (Kent et al. 1991; Fux \& Martinet 1994).

In our model, the NS formation rate outside $500 \mathrm{pc}$ around the Sun corresponds to $\sim 0.884 \times 10^{-11} \mathrm{pc}^{-2} \mathrm{yr}^{-1}$. This rate is lower than the one stated by Tammann et al. (1994). Assuming that the Milky Way is type Sbc, and combining the corresponding value with the historical rate in the Galaxy, Tammann et al. reported a local SN rate of $2 \times 10^{-11} \mathrm{pc}^{-2} \mathrm{yr}^{-1}$. Tammann et al. (1994) gave also a rate of $2.9 \times 10^{-11} \mathrm{pc}^{-2} \mathrm{yr}^{-1}$, which is based on the census of O-B2 stars in $1 \mathrm{kpc}$ around the Sun. Clearly, both values should be dominated by the productive Gould Belt. Outside the Belt the rate should be lower, however, it should be anisotropic and nonuniform on the scale of $3 \mathrm{kpc}$. Our adopted values, $\sim 3.438 \times 10^{-11} \mathrm{pc}^{-2} \mathrm{yr}^{-1}$ inside $500 \mathrm{pc}$ 
and $\sim 0.884 \times 10^{-11} \mathrm{pc}^{-2} \mathrm{yr}^{-1}$ outside $500 \mathrm{pc}$, are chosen to reflect this fact. At the $500 \mathrm{pc}$ transition, our model gives comparable values due to the scaling through $\mathrm{OB}$ associations. We checked that at this transition our model distance distribution of unabsorbed NS show consistently increasing numbers. Probably, we slightly underestimate the NS formation rate at larger distances in the directions of the Galactic center and spiral arms. However, for our calculations closer regions are more important (see distance distributions of ICoNSs below). Anyway, we warn the reader that some uncertainty in the birth rate normalization is hidden inside our model. A comparison of the $\log N-\log S$ distribution, for old and new models of the initial spatial distribution of NSs, is made in Fig. 3 and discussed in Sect. 3.

\subsection{The interstellar absorption of $X$-rays}

$\mathrm{X}$-rays are absorbed by the heavy elements of the interstellar medium. This is especially true for the soft X-rays below $1 \mathrm{keV}$. Their extinction has to be accounted for if one aims at realistic estimates of the sources' observability. The amount of material between the object and the observer is measured as hydrogen column density $N(\mathrm{H})$. Then, assuming the same abundances toward all directions, and accounting for photoelectric absorption processes by atoms, ions, and dust particles, one can determine the X-ray extinction. The treatment of X-ray absorption is significantly upgraded in our new population synthesis, with respect to previous studies, in consideration of all these aspects, i.e., a more realistic model for $N(\mathrm{H})$, up-to-date abundance tables and cross-sections (see Posselt et al. 2007 for a more thorough discussion, and Paper III for details about previous treatment of the ISM $_{\text {absorption }}^{1}$ ).

In the following, we use the abundance tables and photoelectric cross-sections by Wilms et al. (2000) as combined in the tbabs routine, which is implemented, e.g., in XSPEC ${ }^{2}$. We also introduce two different, new $N(\mathrm{H})$ models in three dimensions to account for the highly inhomogeneous distribution of the interstellar medium (ISM). Both models take into account the most updated results in the local neighborhood (up to $230 \mathrm{pc}$ ) by Lallement et al. (2003). Their work well describes the local bubble, which is surrounded by clumps of denser ISM and connected by chimneys to other bubbles. For distances larger than $230 \mathrm{pc}$ we use the analytical ISM model described in Popov et al. (2000) in one of our new $N(\mathrm{H})$ models. In the following, we refer to this model as the "analytical ISM model". For the second $N(\mathrm{H})$ model we use the extinction study by Hakkila et al. (1997). In the following, we refer to this model as the "Hakkila ISM model".

Concerning the transition region at $230 \mathrm{pc}$, we want to make the following short comments. The column density can only increase with larger distances, thus the new data from the analytical formulae/the Hakkila study are only applied if the resulting column densities are larger than those from Lallement et al. (2003) at $230 \mathrm{pc}$. At distances larger than $230 \mathrm{pc}$ the models become naturally much coarser due to fewer measurements. For the analytical model the transition is smooth since the analytical formulae at, e.g., $240 \mathrm{pc}$ result in values similar to the ones at 230 pc by Lallement et al. (2003). In case of the Hakkila

\footnotetext{
1 We also corrected, in the old ISM model, the use of full widths of half maximum instead of the required variances in the exponential density factors by Zane et al. (1995); Dickey \& Lockman (1990). However, the effect of smaller ISM density above the Galactic plane on our model results was quite low.

${ }^{2}$ http://heasarc.gsfc.nasa.gov/docs/xanadu/xspec/
}

model the transition is more abrupt due to the patchiness of the available measurement data applied for the extinction study by Hakkila et al. (1997). On average the hydrogen column density at around $230 \mathrm{pc}$ is around $10^{20} \mathrm{~cm}^{-2}$. This average value increases by around $10^{20} \mathrm{~cm}^{-2}$ at the transition for the Hakkila model, while in case of the analytical model the average increase is only around $10^{19} \mathrm{~cm}^{-2}$.

We checked both models with open cluster extinction data presented by Kharchenko et al. $(2005 \mathrm{a}, \mathrm{b})$ and Piskunov et al. (2006), which are complete up to a distance of 850 pc. We then included the data of the open clusters in both models (see Posselt et al. 2007, for details on the models). We also compared the column densities derived from both ISM models at large distances with the results by Schlegel et al. (1998) from infrared dust emission measurements. In both cases, the analytical ISM model and the Hakkila ISM model, data cubes in Galactic coordinates (steps of one degree) and distance (steps of $10 \mathrm{pc}$ ) are used for our population synthesis. The sampling of the data cube does not reflect the actual accuracy, which is around $25 \mathrm{pc}$ within $230 \mathrm{pc}$ and much coarser at larger distances.

It is noted that both models are large-scale, and even the Hakkila ISM model does not account for small ISM clumps, especially at large distances, above $1 \mathrm{kpc}$.

\subsection{Mass spectrum}

NS cooling curves strongly depend on masses. Once the cooling scenario is chosen, it is therefore necessary to specify the NS's mass spectrum. As in our previous studies, we use the mass spectrum of NSs, which is derived by the joint use of Hipparcos data on spectral classes of close-by massive stars and calculations by Woosley et al. (2002). In addition, in this paper, a modified mass spectrum is studied obtained with the calculations from Heger et al. (2005).

Both mass spectra are obtained in a similar way, and a priori it is impossible to say that the "new" one is preferable with respect to the "old" one. We take spectral types of massive stars around the Sun as given in the Hipparcos catalogue (Perryman \& ESA 1997). To each spectral type we associate a range of masses. Applying now the calculations by Woosley et al. (2002) or, instead, by Heger et al. (2005) we obtain the baryonic masses of compact objects. Then the baryonic masses are translated into gravitational masses with the simple equation, valid for hadronic NSs, by (Timmes et al. 1996)

$$
M_{\mathrm{bar}}-M_{\mathrm{grav}}=0.075 M_{\mathrm{grav}}^{2}
$$

The accuracy of this relation is sufficient for our purposes, as one needs to know the mass within a few percent (less than half the size of a mass bin). Finally, we have a mass spectrum with eight mass bins that corresponds to the eight cooling curves we use in our calculations.

In Fig. 2, we show the two mass spectra, the mass spectrum that was also used in previous studies as well as the new one. Note the nonequal widths of the mass bins. Eight cooling curves for gravitational masses 1.1, 1.25, 1.32, 1.4, 1.48, 1.6, 1.7, and $1.76 M_{\odot}$ are taken, in both the new and old mass spectra. Each bin boundary corresponds to the mean between two values of mass used in calculations.

For the old mass spectrum in Fig. 2, we use the relation between a progenitor mass and NS baryonic mass derived from Fig. 17 by Woosley et al. (2002). We approximate the data in this figure with two linear relations (for progenitor masses $<15 M_{\odot}$ and $>25 M_{\odot}$, respectively), and constant between 15 and 25 solar masses (see Paper II). A peak at $1.4 M_{\odot}$ appears due to the 


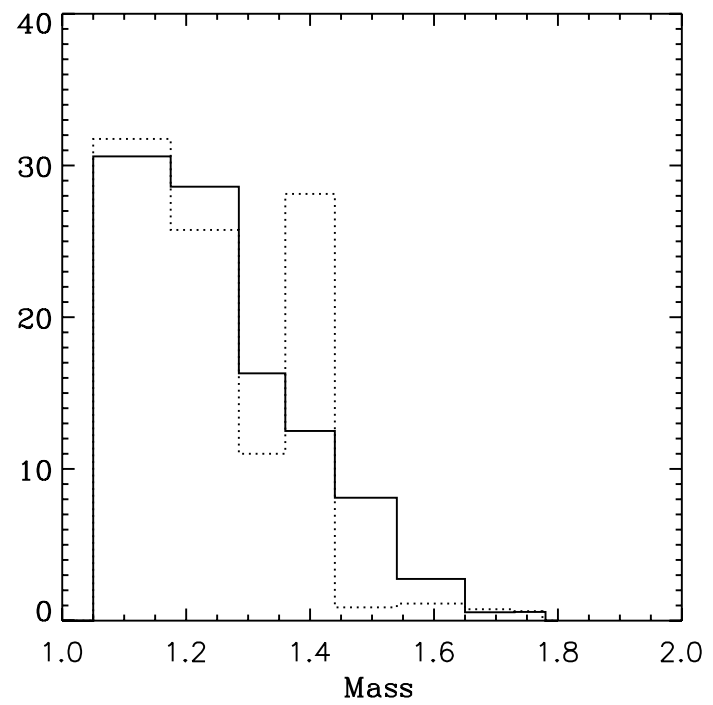

Fig. 2. The old (as used in previous studies, dotted line) and the new (as introduced in this work) mass spectra, binned over eight intervals of different widths (see the text).

fact that in this region progenitors of different mass produce NSs of similar masses. The new mass spectrum, shown with a solid line, is derived much in the same way, but using data from Heger et al. (2005) for stars with masses $>12 M_{\odot}$. As it is visible in the figure, the peak at $1.4 M_{\odot}$ disappears. This is because the dependence of the compact star mass on the progenitor mass contains no flat regions. The most important feature of both mass spectra is a very small number of NSs with masses above $\sim 1.5 M_{\odot}$. As the cooling of a NS is faster for massive stars, a small number of massive objects implies a small number of cold stars in the same age group.

\subsection{Detector properties}

As before (e.g., in Paper I), we use the instrumental response of ROSAT to draw conclusions with respect to the ROSAT All Sky Survey (RASS). However, the energy range considered, e.g. in Paper I was $0.08 \mathrm{keV}$ to $3 \mathrm{keV}$, while the RASS data analysis was done for a slightly smaller energy range. To be in full agreement, we changed the energy range to $0.1 \mathrm{keV}$ to $2 \mathrm{keV}$. The effective area of the instrument is discontinuous over energy due to sharp instrumental absorption edges in the energy band of interest. With respect to previous studies, we improved our sampling of the effective area measurements to better account for such absorption edges. In few cases, we found differences of up to $40 \%$ in the resulting absorbed fluxes for an object. However, this effect is pronounced only for very soft sources at very small distances. Thus the influence on the overall results of the population synthesis are small. We note that, of course, one has to consider vignetting of the X-rays as not all RASS sources are observed on-axis. This is done by an effective area measurement with averaged vignetting applicable for the RASS.

\section{Results of calculations and discussion on the influence of model modifications}

In this section, we compare results of different modifications of the population synthesis scenario studied in this paper. We start by comparing the $\log N-\log S$ distributions calculated with different assumptions, proceed to the resulting map of the cooling

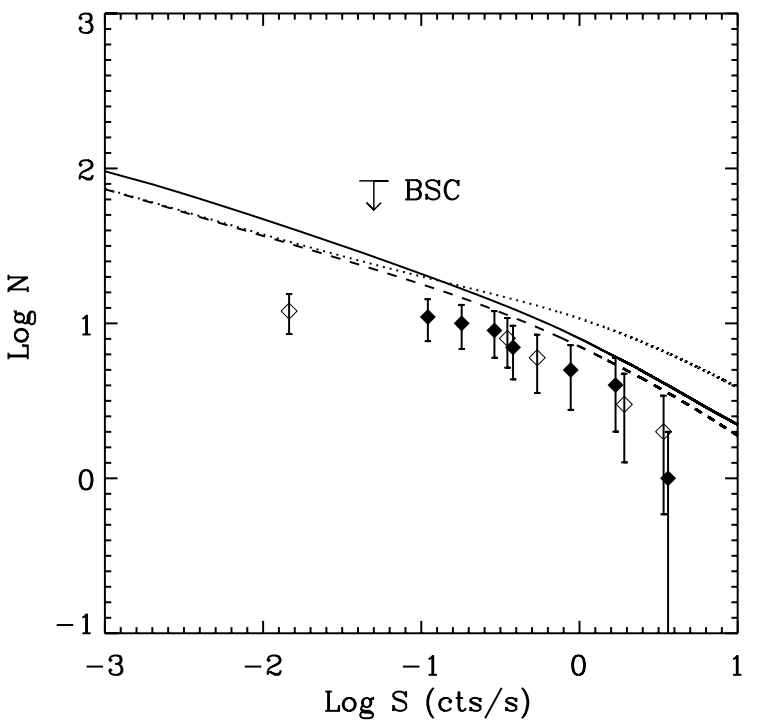

Fig. 3. $\log N-\log S$ for the new initial spatial distribution (solid line) and two variants of the old spatial distribution with $R_{\text {belt }}=300 \mathrm{pc}$ (dotted) and $500 \mathrm{pc}$ (dashed). The curve shown as a solid line is used as a reference on the following plots, too. This curve is calculated for the old mass spectrum, old ISM distribution, and new ISM element abundances. The data points represent the $\log N-\log S$ distribution for the known, young close-by isolated neutron stars with thermal Xray emission (the Magnificent Seven, Geminga, the "second Geminga" - 3EG J1835+5918, and three radio pulsars: B0833-45, B0656+14, B1055-52). Filled symbols correspond to the Magnificent Seven, open symbols to the other NSs. Error bars are plotted for the poissonian errors. We removed PSR1929+10 from the list in Popov et al. (2003) since results of the recent XMM-Newton observations by Becker et al. (2006) demonstrate that the spectrum is non-thermal.

neutron star distribution on the sky, followed by age and distance distribution, and finally discuss the implications on searches for new ICoNS candidates.

\subsection{Comparison of $\log N-\log S$ distributions for different model modifications}

The effects of the successive modifications in our PS-model on the $\log N-\log S$ distribution are shown in Figs. 3-5. The solid curve in all the figures is the same. It corresponds to the new initial spatial distribution of the NSs, new abundances and crosssections, the old mass spectrum, and the old ISM model.

At first, we discuss the influence of the new initial spatial distribution of NSs (i.e. distribution of progenitors). We compare three models: two variants of the old model with $R_{\text {Belt }}=300 \mathrm{pc}$ and $500 \mathrm{pc}$; and the model with the new initial spatial distribution described in Sect. 2.1. $\log N-\log S$ curves are shown in Fig. 3. Except for the initial spatial distribution, all other parameters are the same: old mass spectrum (Fig. 2, dotted curve), new abundances, old ISM distribution (calculations with the new ISM distribution take long times, so for comparison of different modifications we use the old ISM distribution model when possible).

The solid curve corresponds to the new initial spatial distribution. The result with the new progenitor distribution lies in between those of the two old ones for large and moderate fluxes. In fact, our new calculation is only slightly above the simpler model with a Gould Belt radius of 500 pc. For count rates $\log S<-1$, the new initial spatial distribution results in roughly 0.1 dex more observable sources. The new model of the spatial distribution 


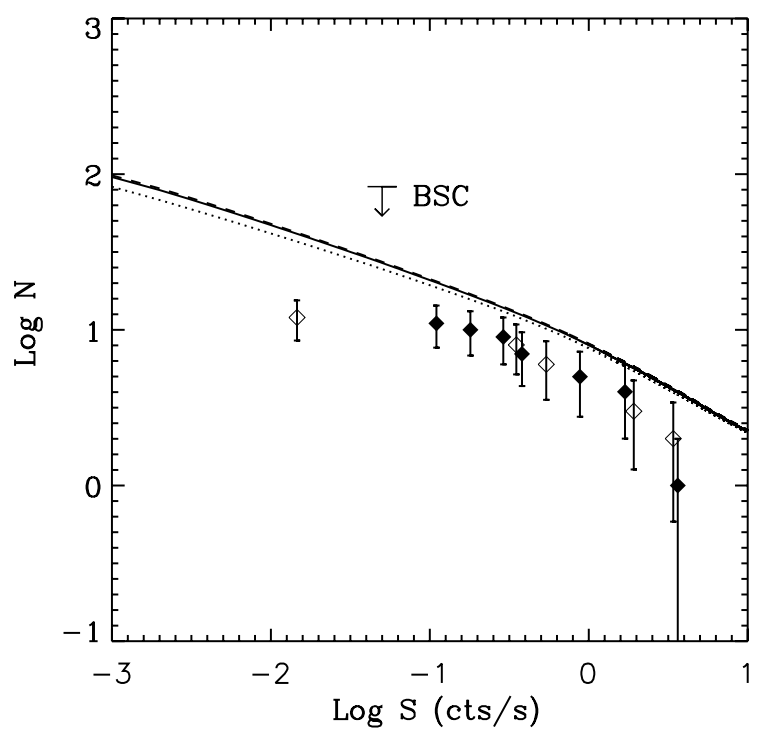

Fig. 4. In this figure, we present the effect of the new mass spectrum and new ISM element abundances. All curves are plotted for the new initial spatial distribution of NSs. Solid curve: old mass spectrum and new abundances. Dotted line: old mass spectrum and old abundances. Dashed curve: new mass spectrum and new abundances. All curves are calculated for the old ISM distribution model.

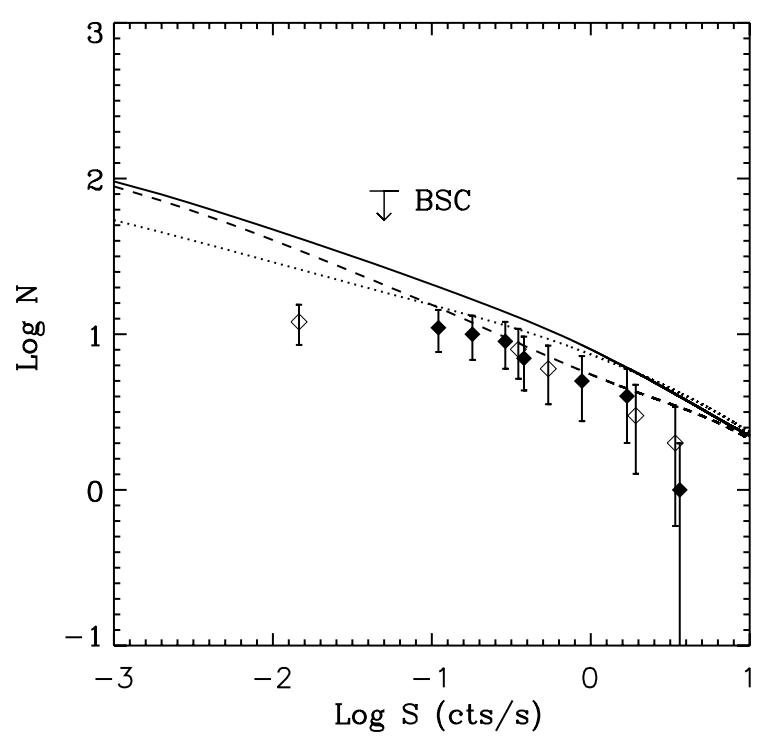

Fig. 5. $\log N-\log S$ in case of different X-ray absorbing ISM models. All curves are plotted for the new initial spatial distribution, the old mass spectrum and new ISM element abundances. Solid curve: old, simple analytical ISM model as, e.g., in Paper III. Dotted curve: new improved analytical ISM model; dashed curve: Hakkila ISM model, see Sect. 2.2.

and the old for $R_{\text {Belt }}=500 \mathrm{pc}$ are in reasonable correspondence with observations at the bright end, where it is assumed that our knowledge about observable objects is mostly complete (as we see below, new models of the ISM distribution shift the curve down, improving the correspondence). However, the locations of observable sources on the sky for the old and new initial spatial distributions are significantly different (compare Fig. 6 below and Fig. 6 in Paper II).

Next, one has to compare the $\log N-\log S$ distributions calculated for different mass spectra (see Sect. 2.3). Computations included the new initial spatial NS distribution, new ISM element abundances and old ISM distribution. Results are shown in Fig. 4. To see the effect of the new mass spectrum, one has to compare the solid and the dashed curves. The solid curve is the same as in Fig. 3. It corresponds to the old mass spectrum (Fig. 2, dotted line). The dashed curve, which is nearly indistinguishable from the solid one, is calculated for the new mass spectrum (Fig. 2, solid line). Surprisingly, the two different mass spectra provide nearly the same result for the $\log N-\log S$. This is very positive for our approach since the mass spectrum is a poorly determined component of the model. As noted above, none of the mass spectra applied here is currently clearly superior. In the following, we continue to use the "old" mass spectrum.

In Fig. 4, the effect of the new ISM element abundances is also shown. The dotted curve should be compared with the solid one. As it can be seen, the effect is not very important. Naturally, it more strongly influences faint sources, which are situated at larger distances (on average). However, even for far away sources, the effect is not significant.

Finally, we compare the $\log N-\log S$ curves obtained for three different models of the ISM distribution. In Fig. 5, we show results for the old simple model (reference curve), the new analytical ISM model, and the Hakkila ISM model. The curve of the new analytical ISM model is very close to the curve of the old ISM model for bright sources. As sources become fainter the number of observable objects becomes smaller than before due to the appearance of directions with high absorption. Using the Hakkila ISM model, we obtain a $\log N-\log S$ curve, which fits the known observational constraints even better. For small fluxes this curve approaches the reference one. In comparison to the analytical ISM cube there are large "windows" of low extinction in the Hakkila ISM model resulting in more sources at low fluxes. The analytical model, on the other hand, seems to overpredict the absorption when going to larger distances (Posselt et al. 2007). Thus, at fainter fluxes one may regard the results of the analytical and Hakkila ISM model as lower and upper limits on the number of observable sources.

We conclude, that in terms of the $\log N-\log S$ distribution our new, more realistic, models are in good agreement with the older ones. The interstellar absorption by the Hakkila ISM model results in a $\log N-\log S$ curve situated slightly closer to the known observations of INSs.

By using a different set of cooling curves one may improve the agreement between computed and observed $\log N-\log S$ distributions. A detailed analysis is, however, outside the scope of the present investigation. Therefore, in the following, only a few comments are made regarding the set of cooling curves applied in Paper III. As apparent from Figs. 3 and 5, our final $\log N-\log S$ curves, which include the new ISM distribution models, are not significantly different from the curve using $R_{\text {belt }}=500$ pc in our old calculations (Paper III). So, the main conclusions about different sets of cooling curves presented in Paper III should not change. While the curve calculated in the case of the Hakkila ISM model - dashed in Fig. 5 - lies at low count rates slightly below the old curve with $R_{\text {belt }}=500$, this deviation is not strong enough to validate the cooling models that we rejected in Paper III. Moreover, for this choice of the ISM distribution, the cooling models, considered as acceptable in Paper III, fit the observations even better.

\subsection{Sky maps}

In earlier papers on population synthesis calculations, we did not attempt to produce realistic sky maps of the ICoNS distribution, as some of our assumptions were quite crude. Now, 


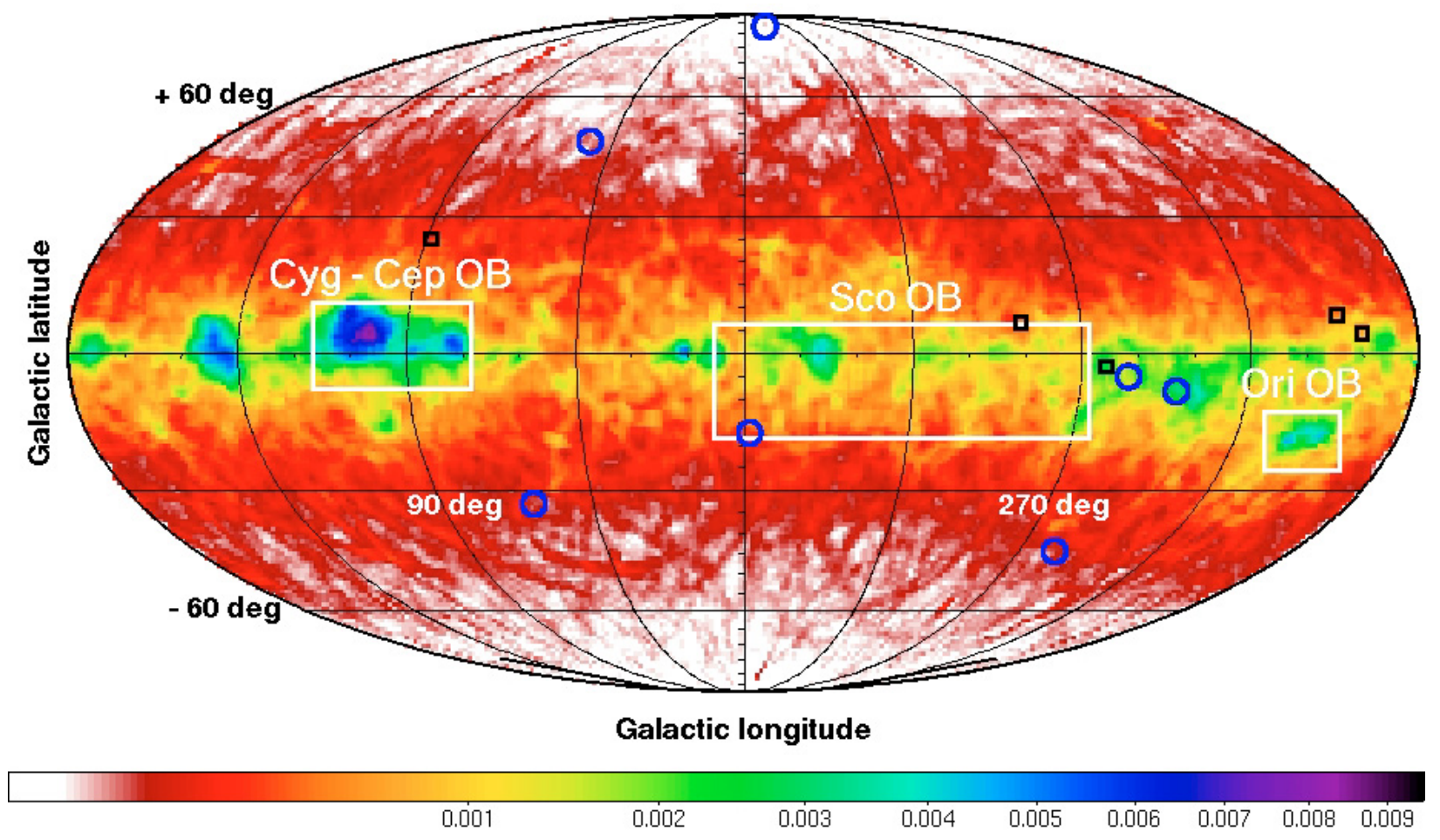

Fig. 6. The expected number density of isolated neutron stars with thermal X-ray emission in units of numbers per square degree. The Galactic map is in Mollweide projection. Only sources with ROSAT PSPC count rates larger than $0.05 \mathrm{cts} \mathrm{s}^{-1}$ are considered, the same value as used in Paper II in Fig. 6. The simulation was done for new initial progenitor distribution, new abundances, old mass spectrum, and the new analytical ISM model, thus corresponding to the dotted $\log N-\log S$ curve in Fig. 5. Marked in blue are the positions of the Magnificent Seven and in black the positions of close young radio pulsars with detected thermal X-ray emission.

with detailed distributions of ISM and birth places of NSs, we can try to compute such maps. In Fig. 6, we present a map in Galactic coordinates for the new initial spatial distribution of NSs, old mass spectrum, and the new analytical ISM model. The plot shows the expected number density for sources with count rates $>0.05 \mathrm{cts} \mathrm{s}^{-1}$. Obviously, sources appear to be restricted toward the Galactic plane and the plane of the Gould Belt. Few objects are expected to be found at latitudes higher than $30^{\circ}$ (see, however, Sect. 3.5). Inside \pm 30 degrees from the Galactic plane the distribution of sources is dominated by NSs from relatively close, rich $\mathrm{OB}$ associations. We mark by boxes the most important of them: Sco OB2, Cyg OB7, Cep OB3, and Ori OB1. Interplay between source distribution and 3D ISM structure allows us to make predictions on which directions are most promising for looking for new ICoNSs.

The computed distribution of sources in Fig. 6 is dominated by sources with count rates in the range $[0.05,0.1] \mathrm{cts} \mathrm{s}^{-1}$. This corresponds to the dimmest sources among identified radio and $\gamma$-ray silent ICoNS, or even to count rates smaller than $<0.1 \mathrm{cts} \mathrm{s}^{-1}$ where there are not currently any ICoNS known. In the distribution of known ICoNSs (Motch et al. 2007), there are no sources with Galactic longitude in the range $\sim 50^{\circ}$ to $\sim 200^{\circ}$. At the first glance, this is in contradiction with the our present map. However, it is necessary to note, that for brighter sources $\left(>0.5\right.$ cts s$\left.^{-1}\right)$ the Cygnus-Cepheus region does not give a strong contribution, and 1-2 objects could escape identification in this overcrowded area close to the Galactic plane (see Sect. 3.4, for a more detailed discussion).

In Fig. 7, we present a similar map for the same parameters concentrated on the faint sources having nominal ROSAT count rates between $0.001 \mathrm{cts} \mathrm{s}^{-1}$ and $0.01 \mathrm{cts} \mathrm{s}^{-1}$. We note that the RASS is not so deep. Figure 7 demonstrates the effect of more distant (>1 kpc) OB associations, with the most important ones marked on the map.

\subsection{Age and distance distributions}

In this paper we introduce age and distance distributions of close-by NSs as predicted by our PS model. These distributions can help to illustrate better the properties of both discovered and still elusive ICoNSs. Figures 8 and 9 show the computed age and distance distributions for NSs with ages up to $10^{6}$ years with bin size $10^{5}$ years, and distances up to $700 \mathrm{pc}$ with bin size of $140 \mathrm{pc}$, calculated for different model assumptions (the two variants of the initial spatial distribution of NSs, and the three models of ISM distributions).

We start by presenting only the general picture and refer to Sect. 3.3.1 for a comparison of the impacts the different PS scenarios have on our results. Figure 8 confirms the natural expectation that the youngest neutron stars contribute most to the observable object fraction in all flux ranges, as they are both brighter and hotter. The radial distribution for different count rates is presented in Fig. 9. While for large fluxes most sources are situated in the region $\sim 200-400$ pc (i.e. in the Gould Belt), for fainter objects the picture is different. At fluxes below $\sim 0.1$ cts s$^{-1}$ one expects to see mostly sources behind the Gould Belt. These general features are very important, as they indicate that new, still unidentified ICoNS are expected to be young objects behind the Gould Belt. We discuss this proposition below in Sect. 3.4. 


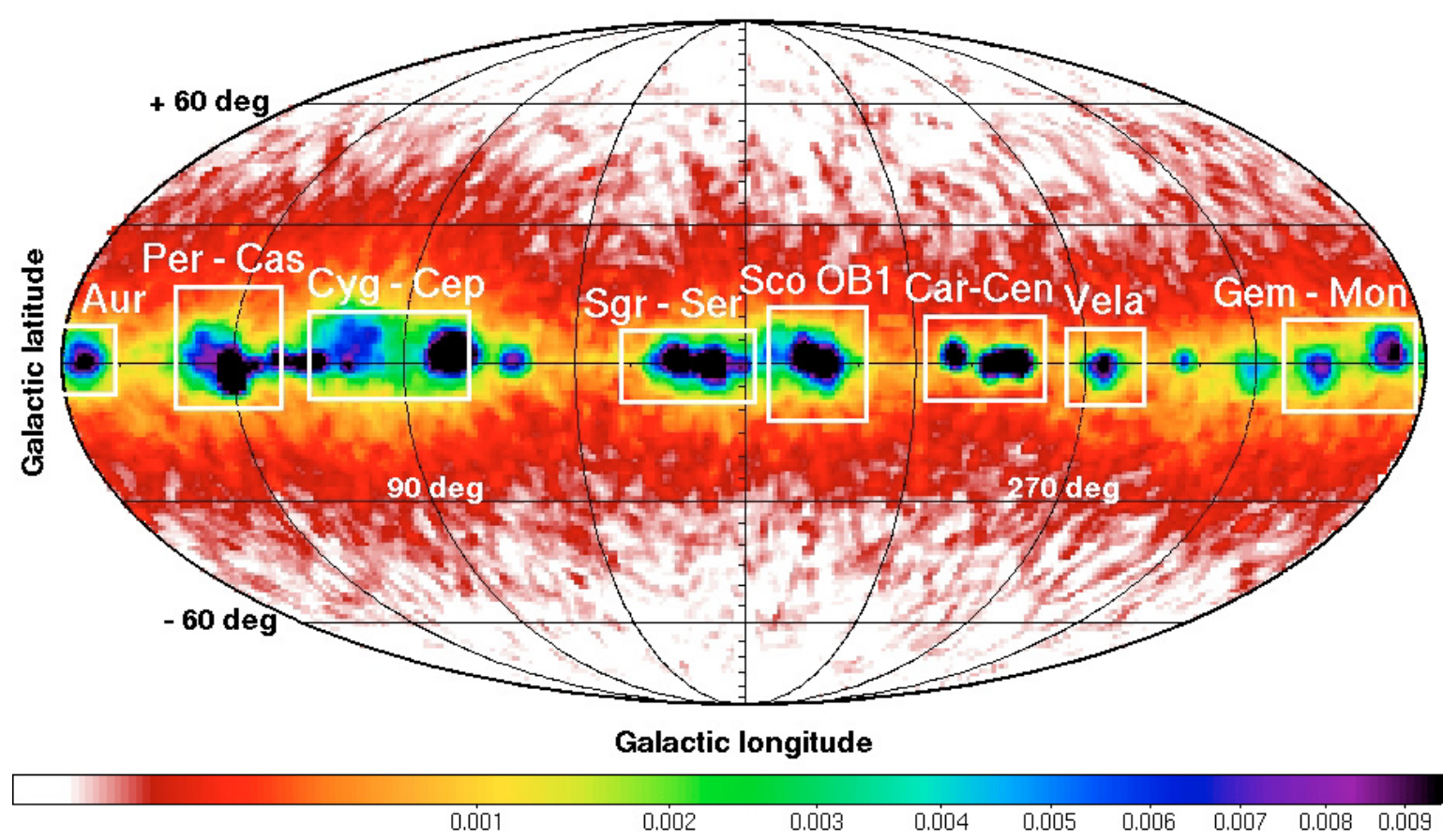

Fig. 7. Same as Fig. 6, but here only faint sources with ROSAT PSPC count rates between $0.001 \mathrm{cts} \mathrm{s}^{-1}$ and $0.01 \mathrm{cts} \mathrm{s}^{-1}$ are considered. Marked are regions of the corresponding $\mathrm{OB}$ associations giving birth to the neutron stars. These OB associations have distances between $1 \mathrm{kpc}$ and $2 \mathrm{kpc}$.

\subsubsection{Comparison of the distributions for the different model modifications}

The population synthesis models used to obtain the results presented in Figs. 8 and 9 differ, on the one hand. in the initial progenitor distribution and, on the other hand, in the adapted ISM model. As it is already evident from the $\log N-\log S$ curve in Fig. 3 that the predicted number of observable NSs is generally higher in the case of the new initial progenitor distribution compared (white bars) to the old one (vertically striped bars). The decrease in number from the youngest to the oldest sources for both progenitor distributions is similar. For sources with count rate $<1 \mathrm{cts} \mathrm{s}^{-1}$, the new initial spatial distribution results in larger number of young sources. These are objects behind the Gould Belt in OB associations. Outside the Gould Belt the new distribution is more compact than the old one: most associations are closer than $2 \mathrm{kpc}$ (in the old model NSs were uniformly distributed in the disk up to $3 \mathrm{kpc}$ ).

As the next step, we compare the results for the old simple ISM model (white bars in Figs. 8 and 9) with those obtained with the new analytical model (black bars). In both cases, we use the new inital progenitor distribution. In general, calculations with the old ISM model produce more observable sources (which can also be seen in the $\log N-\log S$ curves in Fig. 5). This difference is more clearly pronounced for faint sources with count rates below $1 \mathrm{cts} \mathrm{s}^{-1}$ and ages below $3 \times 10^{5}$ years. The X-ray emission of these objects must be more absorbed in the new analytical ISM model to explain this effect. Therefore, these young faint sources have to be located on directions that correspond to larger hydrogen column densities in the new analytical ISM model. Indeed, there are several small regions of higher column density in the Galactic plane, and some of them hinder the view toward star associations, e.g., at $l=215^{\circ}$.

The distance histograms at different count rates at low distances (e.g., up to the third distance bin, [280,420] pc) show no difference or only slight reduction, comparing the resulting numbers obtained by applying the old ISM with those obtained by using the new analytical ISM model. At larger distances in the distance histograms, there are fewer observable NSs in the case of the new analytical model in comparison with the old one, e.g., in the last distance bin, $[560,700] \mathrm{pc}$, in case of the faintest sources. These differences are evident from the properties of the new analytical ISM model. First, due to the inhomogeneties in the new analytical ISM model there are regions of higher column density compared to the old model. Second, the "average" column density (without apparent ISM clumps) in the Galactic plane is lower for the new model at distances below $450 \mathrm{pc}$.

The lower average $N(\mathrm{H})$ value at relatively close distances can explain the comparable or even slightly increased predicted number of observable neutron stars even with present ISM clumps. The ISM clumps are, however, responsable for the number reduction in the right part of the distance histograms. For example, there is strong absorption toward the direction $l=100^{\circ}, b=-5^{\circ}$, relatively close to the Cygnus-Cepheus OBassociations region. The predicted number of observable NSs is reduced there. Since the OB associations toward this direction of sight are at distances larger than 500 pc (e.g., Cyg OB7 at 740 pc, Cep OB2 at 700 pc and Cep OB3 at 900 pc, Cam OB1 at 900 pc) the effect is pronounced mostly at faint count rates (objects relatively far away).

Now we compare the predicted numbers calculated with the analytical ISM model (black bars) and the Hakkila extinction model (diagonal stripes). Here, we note again that up to a distance of $230 \mathrm{pc}$, both ISM models are almost the same. From 240 pc the extinction study by Hakkila et al. (1997) is applied, and so starting from this point the absorption in this more inhomogenous model is usually much larger than in the case of the analytical model, which is characterized by slower and more homogenous increase of the column density. Only at distances around $450 \mathrm{pc}$ do the hydrogen column densities of the analytical ISM model become larger than those of the Hakkila ISM model in most regions. For example, the Hakkila ISM model has, in general, lower hydrogen column densities at $|b|>10^{\circ}$ for 

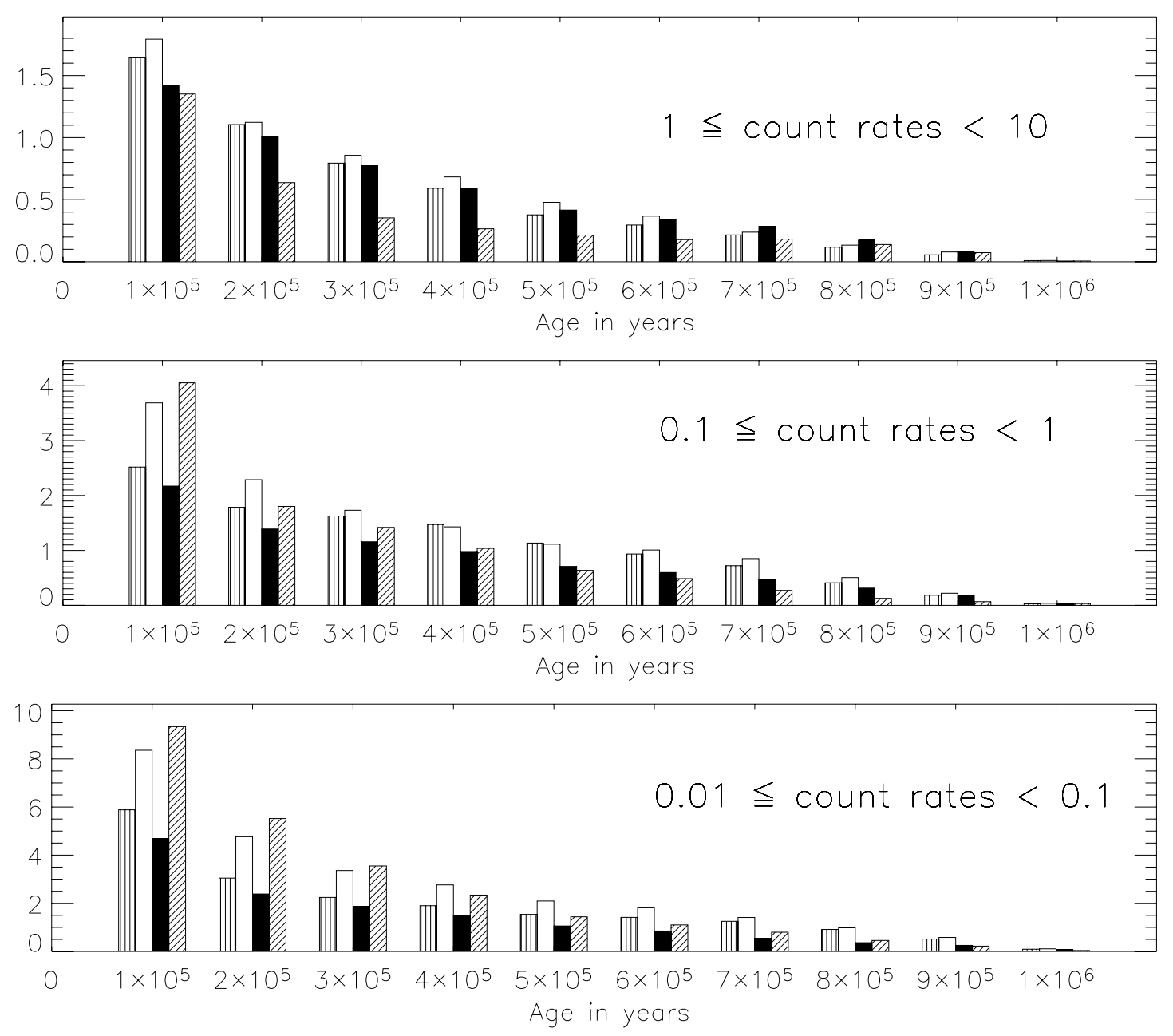

Fig. 8. In this figure, the age histograms of observable INSs with thermal X-ray emission are plotted. Three different ROSAT count rate intervals represent very bright, bright, and moderatly bright ROSAT X-ray sources (note the RASS Bright Source Catalogue limiting count rate of $0.05 \mathrm{cts} \mathrm{s}^{-1}$; Voges et al. 1999). Different bars correspond to different variants of our model. The bars with vertical stripes indicate the results of the old population synthesis model with a Gould Belt radius of 500 pc (Paper II, see also Sect. 2.1), the white bar correponds to the population synthesis applying the new inital spatial distribution (Sect. 2.1, reference $\log N-\log S$ curve in Fig. 3 to Fig. 5). Results obtained in the frame of the new analytical ISM model are shown with black bars, and those obtained with the Hakkila ISM model are represented by bars with diagonal stripes (see Sect. 2.2 and Fig. 5 for the corresponding $\log N-\log S$ curves). Please note different $y$-scales in different panels.

distances larger than $400 \mathrm{pc}$. Extinction is also low in this ISM model from $l=230^{\circ}$ to $l=285^{\circ}$ in the Galactic plane (all distances), where differences of up to an order of magnitude can be reached in comparison to the analytical ISM model.

In agreement with the $\log N-\log S$ curves of Fig. 5 the Hakkila NS number of bright sources is slightly lower than in the case of the analytical model, both in the age and distance histograms. This slightly lower observable NS number is due to larger absorption at closer distances. For count rates below $1 \mathrm{cts} \mathrm{s}^{-1}$, the application of the Hakkila ISM leads to a higher number of observable NSs, especially of young age. The lower absorption at large distances explains these higher observable numbers of ICoNSs. For the discussed distance range, however, the distance histograms indicate only small differences for sources fainter than $1 \mathrm{cts} \mathrm{s}^{-1}$.

\subsection{Resultant search strategies}

The main aim of this study is to make advances in the strategy for searching for new ICoNSs. Based on the results presented above we can suggest some modifications in the approach to look for new candidates. According to what we discussed in the previous sections, new candidates expected to be identified at ROSAT count rates $<0.1 \mathrm{cts} \mathrm{s}^{-1}$ should be young objects born in rich OB associations behind the Gould Belt. Most of the recent studies; such as Agüeros et al. (2006); Chieregato et al. (2005); Rutledge et al. (2003), looked for new candidates far from the Galactic plane. It seems that this is not very promising. Our results indicate that ICoNS should be searched in directions of OB associations such as Cyg OB7 and Cep OB3. On average, new candidates should be slightly hotter than the known ICoNSs, as they are younger.

The absence of sources in the second Galactic quadrant (Motch et al. 2007) can be naturally explained taking into account the properties of close-by OB associations. Most of the associations (Per OB2, Cas-Tau, $\alpha$ Per, Cep OB6) marked in this empty region in Fig. 3 from Motch et al. (2007) have ages that do not favor the appearance of young observable NSs at present times. They are too young ( $<7 \mathrm{Myr}$ ) or too old ( $>25 \mathrm{Myr}$ ), see Table 1 above. As noted above, the strong contribution of the Cygnus-Cepheus region to the expected neutron star number, shown in map Fig. 6, comes especially at low count rates. Thus, we expect that sources with $50^{\circ}<l<200^{\circ}$ would be identified 

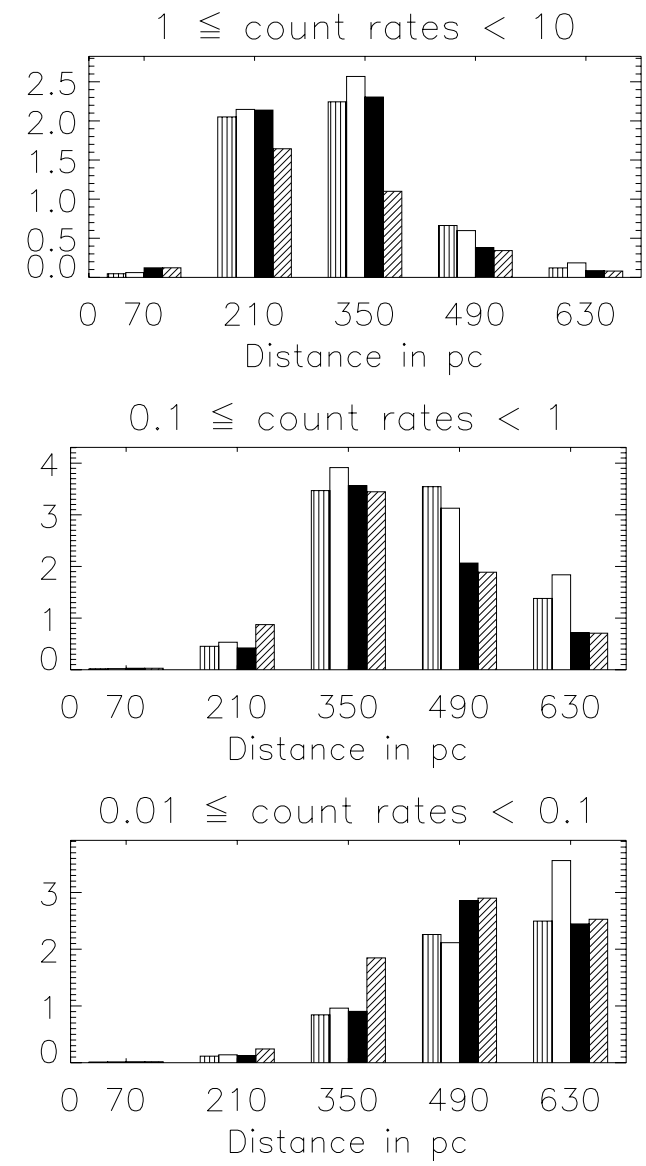

Fig. 9. The distance histograms of the observable INSs with thermal $\mathrm{X}$-ray emission are shown for three different ROSAT count rate intervals. Different bars correspond to different variants of our model, and have the same pattern as in Fig. 8. Please note different $y$-scales in different panels.

having lower fluxes than the known sources, since they come from $\mathrm{OB}$ associations further away.

Considering sky coverage the ROSAT All Sky Survey (RASS) is currently the best choice to look for new ICoNSs in the Cygnus-Cepheus region which is, according to our presented results, the most promising region. However, the relatively large positional error circle of ROSAT usually includes many possible optical counterparts, especially at these low Galactic latitudes. Furthermore, one has to exclude variable $\mathrm{X}$-ray sources to find ICoNSs. In this respect, the recentlypublished XMM-Newton Slew Survey (EPIC with medium filter, e.g. Esquej et al. 2006) may become an important database. Currently, this survey $(0.2-12 \mathrm{keV}$ band) covers roughly $15 \%$ of the sky, and below $2 \mathrm{keV}$ its sensitivity is comparable to that of the RASS (Freyberg et al. 2006). The sensitivity is, however, strongly inhomogeneous perpendicular to the slew direction. The astrometric accuracy is around 17" (90\% confidence level), but in few cases an error of $1^{\prime}$ due to an attitude problem has been reported $^{1}$. In conjunction with the RASS, the XMM-Newton Slew Survey, with its increasing coverage, can be used to identify non-variable X-ray sources and eventually improve their positional accuracy. With the planned expanded energy range down to $150 \mathrm{eV}$ (M. Freyberg, pers. comm.) the soft X-rays emitted by ICoNSs could be detected. However, the XMM-Newton Slew

\footnotetext{
1 http://xmm.esac.esa.int/external/xmm_science/ slew_survey/xmmsl1_ug.shtml
}

Survey has strong inhomogeneous sensitivity, and the RASS is not deep enough for the expected new faint sources. Positions of both can be too inaccurate in the highly-populated Galactic plane. Pointed observations by XMM-Newton, with its high sensitivity, and by Chandra, with its superb positional accuracy, may solve these problems for particular directions.

\subsection{Outlook}

Whether the calculated $\log N-\log S$ curves are realistic descriptions of the ICoNS numbers also at low count rates could be best tested with the help of an All Sky Survey like that with the planned eROSITA ${ }^{2}$. This experiment is expected to have an angular resolution of $<25^{\prime \prime}$ and to be 30 times more sensitive than the RASS (Predehl et al. 2006). eROSITA's CCD technology is based on an improved concept of the successful XMM-Newton EPIC-pn CCDs. While the noise at low X-ray energies is expected to be much lower compared to the EPIC-pn CCDs (Meidinger et al. 2006), the influence of the planned eROSITA filter actually reduces the sensitivity to or below that of ROSAT in case of energies lower than $0.2 \mathrm{keV}$ (P. Predehl, pers. comm.). Using a preliminary eROSITA response matrix, we can estimate that detections of soft blackbody spectra are possible with approximately one eighth of the RASS count rate after the planned 4 years of survey observations. Thus, in principle, sources could be expected to reach count rates down to $\log S \approx-2.7$ (with 0.017 counts s$^{-1}$ as the RASS limit). This can result in a significant increase in the number of currently known ICoNSs, up to an order of magnitude. However, the actual number of identified NSs will depend strongly on the finally reached positional accuracy, especially in the Galactic plane. For finding new sources of the Magnificent Seven type, it will be advantageous to have $\mathrm{X}$-ray positions with $\sim 1^{\prime \prime}$ accuracy to avoid confusion with faint extragalactic objects (Trümper 2006).

Besides the standard approach, based on X-ray/optical wavelengths, we mention below other possibilities to look for ICoNSs. They are not directly related to our PS results. Keeping the restrictions of our model in mind, however, they may be important in terms of finding new ICoNSs.

Recently, Crawford et al. (2006) found 56 well-determined EGRET error boxes devoided of radio pulsars. Estimates of the number of young NSs that can manifest themselves as $\gamma$-ray sources show that many of the 56 sources can be related to these compact objects, but a significant fraction of them should not appear as standard radio pulsars (Harding et al. 2007; Gonthier et al. 2007). Depending on the $\gamma$-ray emission model, between $30 \%$ and $90 \%$ of $\gamma$-ray pulsars may be Geminga-like objects, according to Harding et al. (2007). They can be detectable in soft $\mathrm{X}$-rays as coolers, elusive in the radio band ${ }^{3}$. New GLAST and AGILE observations can provide much smaller error boxes, so soon it could be possible to identify sources if they are ICoNSs. It would be fascinating to find more " $\gamma$-ray selected" coolers. Of course, it is important to make a joint population synthesis for thermal and non-thermal X-ray sources, but this task is out of the scope of this paper.

Another possibility to find new ICoNSs is to search for (un)bound compact companions of OB runaway stars. More than one hundred OB runaway stars are known in a $1 \mathrm{kpc}$ region around the Sun (de Zeeuw et al. 1999). In comparison with

\footnotetext{
2 www.mpe.mpg.de/erosita/MDD-6.pdf

3 Geminga itself was claimed to be detected in radio Malofeev \& Malov (1997), but the "second Geminga" - 3EG J1835+5918 - is observed only in $\gamma$ and in soft X-rays.
} 
typical OB stars they are characterized by large spatial velocities or/and by large distances from the Galactic plane. Two main origins of these large velocities are currently discussed: dynamical interaction and expulsion of a companion in a close binary system (e.g., Blaauw 1961). The latter case is interesting for the discussion of a search for new close-by cooling NSs.

A binary can survive after the first $\mathrm{SN}$ explosion in roughly 10-20\% of cases (e.g., Popov \& Prokhorov 2006). After this event, a runaway system consisting of an OB star and a compact object (most probably a NS) is expected. A young NS can appear as a radio pulsar. Sayer et al. (1996) and Philp et al. (1996) searched for radio pulsar companions of $\sim 40$ runaway OB stars. Nothing was found. This result is consistent with the assumption that in less than $20 \%$ of the cases, OB stars have radio pulsar companions. Still, it is interesting to speculate that runaway massive stars can have ICoNS as companions. Then a companion can be identified as a source of additional X-ray emission if a NS is younger than $\sim 10^{6} \mathrm{yr}$. It is expected to have a thermal component with $T \sim 50-200 \mathrm{eV}$ and $L \sim 10^{30}-10^{32} \mathrm{erg} \mathrm{s}^{-1}$ in most standard scenarios of cooling (see Blaschke et al. 2004; Page \& Reddy 2006; Page et al. 2007, and references therein). Our simple estimates show that a cooling NS in a binary with an OB star can appear in the propeller stage. In this case, one expects additional energy output unrelated to the surface thermal emission.

As mentioned above, in our calculations very few NSs can reach high Galactic latitudes. However, in our model we do not take into account that progenitors themselves can be high velocity objects (runaway stars or so-called hyper-velocity stars, Brown et al. 2005). In this case a NS can be born far away from the Galactic plane. This possibility is important in connection with the recently discovered source, Calvera (Rutledge et al. 2008), which could have had a high velocity progenitor. Recent strict bounds on the radio emission from Calvera (Hessels et al. 2007) make it probable that this source is similar to the Magnificent Seven.

Hyper-velocity stars (Brown et al. 2007) are expected to originate mainly from the Galactic center. They acquire large velocities after binary disruption in the field of the supermassive Galactic black hole. Another possibility is the ejection from young star clusters (Gvaramadze et al. 2007). Initial velocities are about $1000-3000 \mathrm{~km} \mathrm{~s}^{-1}$. In $25 \mathrm{Myr}$ (maximum lifetime of a NS progenitor) a hyper-velocity star can travel up to $50 \mathrm{kpc}$, more than enough to explain Calvera. However, the number of such stars is not high. The total rate of production of hyper-velocity stars of all masses is between $10^{-3}$ and $10^{-6} \mathrm{yr}^{-1}$ (Brown et al. 2007). If we take this rate as $\sim 10^{-6} \mathrm{yr}^{-1}$ for NS progenitors, then one can expect a few ICoNS on very high Galactic latitudes. In this case it is not very probable to find one Calvera in $\sim 10 \mathrm{kpc}$ from us. Still, this possibility cannot be excluded. The high velocity tail of runaway stars corresponds to 200-300 $\mathrm{km} \mathrm{s}^{-1}$. In $25 \mathrm{Myr}$, a NS progenitor can reach $z \sim 5 \mathrm{kpc}$ above the Galactic plane. Potentially, it is enough to explain Calvera, but detailed modeling is desirable.

Of course population synthesis models, in general, and the one we apply in particular, cannot predict new types of sources, since our results do not include populations of coolers absolutely different from those assumed in the model. For example, Calvera could be an evolved version of the Cas A central compact object (CCO, for more information on CCOs see, e.g., Kaspi et al. 2006). Our model does currently not include objects like this (small emitting area, etc.). In general, the differences in properties between CCOs and close-by coolers indicate that we currently do not understand initial properties (and perhaps, evolution) of NSs well enough.

A joint population synthesis of all known types of NSs would be useful. The presented population synthesis model cannot take into account all interesting possibilities related to evolution of cooling NSs. So, as usual, surprises are not excluded.

\section{Conclusions}

In this paper we presented our new, more advanced model for the population synthesis of close-by cooling NSs. The two, sligthly different, mass distributions we consider result in nearly the same observable number of NSs. Detailed treatment of the initial spatial distribution of NS progenitors and a detailed ISM structure up to $3 \mathrm{kpc}$ allow us to discuss the strategy of looking for new ICoNS. Our main results in this respect are the following: new candidates are expected to be identified behind the Gould Belt, in directions to rich OB associations, in particular in the Cygnus-Cepheus region; new candidates, on average, are expected to be hotter than the known population of ICoNS. Besides the usual approach (looking for soft X-ray sources), the search in "empty" $\gamma$-ray error boxes or among run-away OB stars may yield new X-ray thermally-emitting NS candidates.

Acknowledgements. We thank D. Blaschke, H. Grigorian, and D. Voskresensky for data on cooling curves and discussions; A. Mel'nik for discussion of properties of OB associations; R. Lallement for the sodium data; and A. Pires for discussions about the ISM model. The authors thank the anonymous referee for careful reading of the manuscript and the suggested improvements.

S.B.P. was supported by INTAS and Dynasty foundations. This research has made use of SAOImage DS9, developed by Smithsonian Astrophysical Observatory; the SIMBAD and VizieR databases, operated at CDS, Strasbourg, France; and NASA's Astrophysics Data System Bibliographic Services.

\section{References}

Agüeros, M. A., Anderson, S. F., Margon, B., et al. 2006, AJ, 131, 1740 Aguilera, D. N., Pons, J. A., \& Miralles, J. A. 2007, ArXiv e-prints, 710 Aguilera, D. N., Pons, J. A., \& Miralles, J. A. 2008, ApJ, 673, L167

Arzoumanian, Z., Chernoff, D. F., \& Cordes, J. M. 2002, ApJ, 568, 289

Becker, W., Kramer, M., Jessner, A., et al. 2006, ApJ, 645, 1421

Blaauw, A. 1961, Bull. Astron. Inst. Netherlands, 15, 265

Blaes, O., \& Madau, P. 1993, ApJ, 403, 690

Blaha, C., \& Humphreys, R. M. 1989, AJ, 98, 1598

Blaschke, D., Grigorian, H., \& Voskresensky, D. N. 2004, A\&A, 424, 979

Brown, W. R., Geller, M. J., Kenyon, S. J., \& Kurtz, M. J. 2005, ApJ, 622, L33 Brown, W. R., Geller, M. J., Kenyon, S. J., Kurtz, M. J., \& Bromley, B. C. 2007, ApJ, 660, 311

Chieregato, M., Campana, S., Treves, A., et al. 2005, A\&A, 444, 69

Crawford, F., Roberts, M. S. E., Hessels, J. W. T., et al. 2006, ApJ, 652, 1499

Dambis, A. K., Mel'nik, A. M., \& Rastorguev, A. S. 2001, Astron. Lett., 27, 58 de Zeeuw, P. T., Hoogerwerf, R., de Bruijne, J. H. J., Brown, A. G. A., \& Blaauw, A. 1999, AJ, 117, 354

Dickey, J. M., \& Lockman, F. J. 1990, ARA\&A, 28, 215

Esquej, M. P., Altieri, B., Bermejo, D., et al. 2006, in The X-ray Universe 2005, ed. A. Wilson, ESA SP-604, 965

Faucher-Giguère, C.-A., \& Kaspi, V. M. 2006, ApJ, 643, 332

Freyberg, M. J., Altieri, B., Bermejo, D., et al. 2006, in The X-ray Universe 2005, ed. A. Wilson, ESA SP-604, 913

Fritze-v. Alvensleben, U. 2000, in Stars, Gas and Dust in Galaxies: Exploring the Links, ed. D. Alloin, K. Olsen, \& G. Galaz, ASP Conf. Ser., 221, 179

Fux, R., \& Martinet, L. 1994, A\&A, 287, L21

Gonthier, P. L., Story, S. A., Clow, B. D., \& Harding, A. K. 2007, Ap\&SS, 309, 245

Grenier, I. A. 2000, A\&A, 364, L93

Gvaramadze, V. V., Gualandris, A., \& Portegies Zwart, S. 2007, ArXiv Astrophysics e-prints

Haberl, F. 2007, Ap\&SS, 308, 181

Haberl, F., Turolla, R., de Vries, C. P., et al. 2006, A\&A, 451, L17

Hakkila, J., Myers, J. M., Stidham, B. J., \& Hartmann, D. H. 1997, AJ, 114, 2043

Harding, A. K., Grenier, I. A., \& Gonthier, P. L. 2007, Ap\&SS, 309, 221 
Heger, A., Woosley, S. E., \& Spruit, H. C. 2005, ApJ, 626, 350

Hessels, J. W. T., Stappers, B. W., Rutledge, R. E., Fox, D. B., \& Shevchuk, A. H. 2007, A\&A, 476, 331

Hobbs, G., Lorimer, D. R., Lyne, A. G., \& Kramer, M. 2005, MNRAS, 360, 974

Kaspi, V. M., Roberts, M. S. E., \& Harding, A. K. 2006, Isolated neutron stars (Compact stellar X-ray sources), 279

Kent, S. M., Dame, T. M., \& Fazio, G. 1991, ApJ, 378, 131

Kharchenko, N. V., Piskunov, A. E., Röser, S., Schilbach, E., \& Scholz, R.-D. 2005a, A\&A, 440, 403

Kharchenko, N. V., Piskunov, A. E., Röser, S., Schilbach, E., \& Scholz, R.-D. 2005b, A\&A, 438, 1163

Lallement, R., Welsh, B. Y., Vergely, J. L., Crifo, F., \& Sfeir, D. 2003, A\&A, 411,447

Malofeev, V. M., \& Malov, O. I. 1997, Nature, 389, 697

Meidinger, N., Andritschke, R., Hälker, O., et al. 2006, in High Energy, Optical, and Infrared Detectors for Astronomy II, ed. D. A. Dorn, \& A. D. Holland, Proc. SPIE, 6276

Mel'nik, A. M., \& Efremov, Y. N. 1995, Astron. Lett., 21, 10

Miyamoto, M., \& Nagai, R. 1975, PASJ, 27, 533

Motch, C., Pires, A. M., Haberl, F., \& Schwope, A. 2007, Ap\&SS, 308, 217

Page, D., \& Reddy, S. 2006, Ann. Rev. Nucl. Part. Sci., 56, 327

Page, D., Geppert, U., \& Küker, M. 2007, Ap\&SS, 308, 403

Perryman, M. A. C., \& ESA. 1997, The HIPPARCOS and TYCHO catalogues. Astrometric and photometric star catalogues derived from the ESA HIPPARCOS Space Astrometry Mission (Noordwijk, Netherlands: ESA Publications Division), ESA SP Series, 1200

Philp, C. J., Evans, C. R., Leonard, P. J. T., \& Frail, D. A. 1996, AJ, 111, 1220

Pichardo, B., Martos, M., \& Moreno, E. 2004, ApJ, 609, 144

Piskunov, A. E., Kharchenko, N. V., Röser, S., Schilbach, E., \& Scholz, R.-D. 2006, A\&A, 445, 545

Popov, S., Grigorian, H., Turolla, R., \& Blaschke, D. 2006, A\&A, 448, 327 (Paper III)

Popov, S. B., \& Prokhorov, M. E. 2004, [arXiv: astro-ph/0411792]

Popov, S. B., \& Prokhorov, M. E. 2006, MNRAS, 367, 732
Popov, S. B., Colpi, M., Prokhorov, M. E., Treves, A., \& Turolla, R. 2000, ApJ, 544, L53

Popov, S. B., Colpi, M., Prokhorov, M. E., Treves, A., \& Turolla, R. 2003, A\&A, 406, 111 (Paper I)

Popov, S. B., Turolla, R., Prokhorov, M. E., Colpi, M., \& Treves, A. 2005, Ap\&SS, 299, 117 (Paper II)

Pöppel, W. 1997, Fundamentals of Cosmic Physics, 18, 1

Posselt, B., Popov, S. B., Haberl, F., et al. 2007, Ap\&SS, 308, 171

Predehl, P., Hasinger, G., Böhringer, H., et al. 2006, in Space Telescopes and Instrumentation II: Ultraviolet to Gamma Ray, ed. M. J. L. Turner, \& G. Hasinger, Proc. SPIE, 6266

Rutledge, R. E., Fox, D. W., Bogosavljevic, M., \& Mahabal, A. 2003, ApJ, 598, 458

Rutledge, R. E., Fox, D. B., \& Shevchuk, A. H. 2008, ApJ, 672, 1137

Sayer, R. W., Nice, D. J., \& Kaspi, V. M. 1996, ApJ, 461, 357

Schlegel, D. J., Finkbeiner, D. P., \& Davis, M. 1998, ApJ, 500, 525

Schwope, A. D., Hambaryan, V., Haberl, F., \& Motch, C. 2005, A\&A, 441, 597

Takatsuka, T., \& Tamagaki, R. 2004, Prog. Theor. Phys., 112, 37

Tammann, G. A., Loeffler, W., \& Schroeder, A. 1994, ApJS, 92, 487

Timmes, F. X., Woosley, S. E., \& Weaver, T. A. 1996, ApJ, 457, 834

Torra, J., Fernández, D., \& Figueras, F. 2000, A\&A, 359, 82

Trümper, J. 2006, in The Scientific Requirements for Extremely Large Telescopes, ed. P. Whitelock, M. Dennefeld, \& B. Leibundgut, IAU Symp., 232, 236

Trümper, J. E., Burwitz, V., Haberl, F., \& Zavlin, V. E. 2004, Nucl. Phys. B Proc. Suppl., 132, 560

Voges, W., Aschenbach, B., Boller, T., et al. 1999, A\&A, 349, 389

Walter, F. M., \& Matthews, L. D. 1997, Nature, 389, 358

Wilms, J., Allen, A., \& McCray, R. 2000, ApJ, 542, 914

Woosley, S. E., Heger, A., \& Weaver, T. A. 2002, Rev. Mod. Phys., 74, 1015

Yakovlev, D. G., \& Pethick, C. J. 2004, ARA\&A, 42, 169

Zane, S. 2007, Ap\&SS, 308, 259

Zane, S., Turolla, R., Zampieri, L., Colpi, M., \& Treves, A. 1995, ApJ, 451, 739

Zavlin, V. E. 2007, [arXiv: astro-ph/0702426] 